\title{
Buckling with equatorial mattress suture in retinal detachment
}

\author{
MOHAMED AYOUB \\ Department of Ophthalmology, Faculty of Medicine, Cairo Lniversity, Egypt
}

Buckling procedures are useful in securing successful re-attachment of the retina. Apart from their role in ensuring a contact between the retina and the choroid during the postoperative healing period, they also help to relax the pull exerted by the vitreous on the retina. When the traction exerted by the vitreous on the retina is marked, it is desirable to aim at a permanent buckling by the use of a circling element; in other cases a localized buckling procedure may be adopted. In the latter condition, when a firm healing between the retina and the choroid occurs around the breaks, a subsequent gradual flattening of the buckle does not seem to endanger the final result of the operation.

The technique described below is a simple procedure which can effect an adequate $\overrightarrow{6}$ buckling which persists for a few weeks after operation, by which time a firm choroido- N retinal adhesion is produced. It is most suitable for treating detachments involving only one quadrant of the retina, with one or a few small breaks (preferably holes) at one site.

\section{Procedure}

A semicircular incision is made in the conjunctiva and Tenon's capsule. Traction sutures are passed under the three rectus muscles in the locality. The hole is treated with transscleral diathermy or freezing in the usual way. The most posterior coagulation is marked on the sclera with gentian violet and its distance from the limbus is measured. A mattress suture of mersilene $3 / 0$ is placed $\mathrm{I} \mathrm{mm}$. behind the mark in a plane parallel to the equator of the globe (Fig. I). The suture must be one third to one half as long as the circumference of the equator. The suture is fixed to the sclera at each end by two wide stitches where the needle is passed in a direction parallel to the equator. The stitches must be 2 to $3 \mathrm{~mm}$. long and fairly deep, and for this reason they should not be placed under the rectus muscles where the sclera is thin. The hole must lie just anterior to the centre of the suture. After the release of the subretinal fluid, the suture is tightened temporarily and the relation of the break to the buckle is checked by ophthalmoscopy. Usually the buckle is most prominent midway between the two points of fixation of the mattress suture, and the hole should be lying on the anterior slope of the buckle (Fig. 2). When necessary the site of the suture may be modified to obtain the correct position. The suture is now tightened to create an intraocular pressure of $25 \mathrm{~mm} . \mathrm{Hg}$.

\section{Postoperative appearance}

The buckle produced by the mattress suture remains prominent for 3 weeks and thereafter starts to flatten in a gradual manner, until the end of the 6 th week when no trace of it can be detected. This gradual flattening is probably due to the gradual cutting of the stitches at each end of the suture. This was not verified surgically because there was no recurrence in the ten cases of retinal detachment in which the technique was used. 


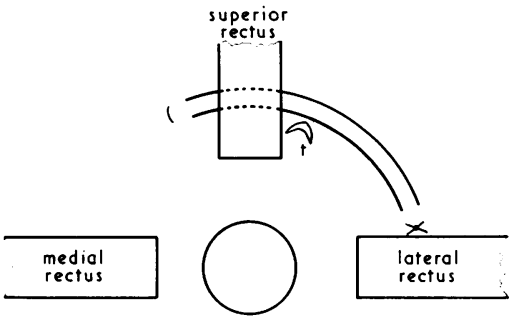

FIG. I Position of mattress suture for a tear $(t)$ in the left eye in the 1 o'clock meridian

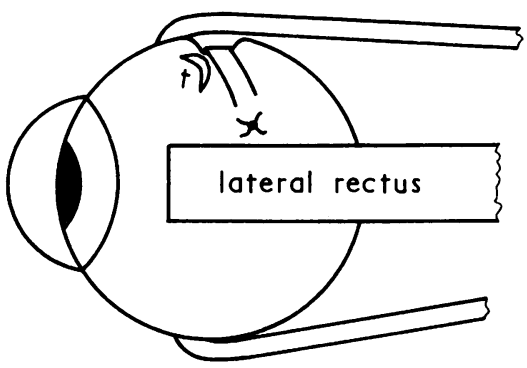

FIG. 2 Lateral view of the same case

\section{Discussion}

Buckling with a mattress suture is a simple method of holding the retina and choroid together for a sufficient time after an operation for retinal detachment. The buckle thereby produced is a temporary one, but it flattens gradually and the elevation disappears after the choroido-retinal adhesion is firm. Good results are to be expected if the procedure is employed in suitable cases, i.e. those with a localized detachment with small breaks (preferably holes) confined to one quadrant of the retina. It should not be used in cases showing gross evidence of vitreous traction, in which more complicated procedures become desirable.

The procedure was employed in ten cases of retinal detachment, in all of which a successful re-attachment was achieved. In two of the treated cases the detachment involved more than two quadrants of the retina, and in one case a horseshoe tear ( 3 disc diameters) with a pulled-in operculum was present.

\section{Summary}

A temporary buckling effect can be produced by placing a mattress suture parallel to the equator of the globe. This simple procedure can assist the successful re-attachment of the retina in selected cases of retinal detachment. 